\title{
EDITORIAL
}

\section{Addressing uncertainty: what is the role of consensus in end-of-life care?}

\author{
Ann C. Long ${ }^{1 *}$, Peter E. Spronk ${ }^{2}$ and Charles L. Sprung ${ }^{3}$
}

๑) 2016 Springer-Verlag Berlin Heidelberg and ESICM

Uncertainty is a familiar companion in the practice of medicine, and this lack of certainty is abundant in endof-life care. Medical decision-making at the end of life occurs in a complex milieu of personal values and beliefs, where tensions between patient autonomy, sanctity of life, quality of life, and social justice abound. Efforts to attenuate this uncertainty through rigorous scientific investigation may be met with legal and ethical challenges, and high-quality evidence is often absent. Without high-quality evidence to guide decision-making, the importance of consensus in end-of-life care becomes readily apparent. Although it may be impractical to establish a universal understanding of what is "beneficial" or what it means to "do no harm" at the end of life, achievement of consensus is possible in many different areas of end-of-life care [1]. When driven by a desire to improve the quality of dying and death for patients and their family members, consensus in end-of-life care can serve as an important source of direction for clinicians. In an article recently published in Intensive Care Medicine, Downar and colleagues [2] take on the challenging task of achieving consensus for the process of withdrawal of life-sustaining treatments.

Using input from an interdisciplinary group of ICU care providers from the Canadian Critical Care Society and the Canadian Association of Critical Care Nurses, the authors employed a modified Delphi process to make recommendations about the withdrawal of life-sustaining treatments in the intensive care unit (ICU). Key issues related to the withdrawal of life-sustaining treatments were identified through review of the literature and then organized into four broad categories: (1) preparation for

\footnotetext{
*Correspondence: along11@uw.edu

${ }^{1}$ Division of Pulmonary and Critical Care Medicine, Harborview Medical Center, University of Washington, 325 Ninth Avenue, Box 359762, Seattle, WA 98104, USA

Full author information is available at the end of the article
}

withdrawal; (2) assessment of distress; (3) pharmacologic management of distress; and (4) the process of withdrawal of life-sustaining therapies [2]. Statements were then generated for each of these categories and a level of recommendation was assigned for each statement, using a unique grading system designed by the authors. During the Delphi process, individual statements were assembled into paragraphs organized by theme. A threshold of $70 \%$ agreement was set for acceptance of each paragraph, and consensus was achieved for all paragraphs during round 3 of the Delphi process. Recommendations address the care and communication provided to patients and their family members before, during, and after the withdrawal of life-sustaining treatments [2].

In their review of the literature, the authors found very little high-quality scientific evidence to support specific practices in the withdrawal of life-sustaining treatments [2]. Does this lack of high-quality scientific evidence affect the importance of these guidelines? Not necessarily. Scientific evidence that fails to reach the benchmark of "high quality" is not without merit, and multiple studies of low or even very low quality can be used to make recommendations after careful consideration of the desirable and undesirable effects of the practice in question. In addition, experiential evidence is also quite valuable and can be particularly helpful when the ability to answer a key question using traditional research methods is limited. In situations where high-quality scientific evidence may be lacking, as in certain domains of end-oflife care, existing scientific evidence and expert opinion can be used to generate consensus about best practices. However, when considering complex topics such as the withdrawal of life-sustaining treatments, achieving consensus may not be a straightforward process. Decisionmaking about end-of-life care may vary widely from provider to provider, with practice patterns influenced by personal values, religion, and culture [3-7]. Although

\section{Springer}


this variability may make consensus in end-of-life care difficult to achieve, the process of achieving consensus is valuable in and of itself. Efforts to achieve consensus will reveal areas of agreement and disagreement, and both can be informative. Achieving and formulating consensus can establish a standard of practice for end-of-life care, while a lack of consensus may highlight areas ripe for future research [1]. Of course, recommendations made from consensus based on low-quality evidence or expert opinion are unlikely to be universally accepted, and disagreement with such recommendations should be respected.

Just as specific standards apply to the conduct of randomized trials and observational studies, the approach to achieving consensus should also be marked by attention to detail and rigor. The process should be iterative, and the methods employed to achieve consensus should be meticulously described with special attention given to the criteria used to determine agreement. Participants should include healthcare providers with extensive clinical experience who understand the nuances of the issues being addressed. When recommendations may have both ethical and legal implications, as is the case with guidelines addressing the withdrawal of life-sustaining treatments, the panel of experts should include ethicists and those with legal expertise. Downar and colleagues were systematic in their approach to achieving consensus, and they did an excellent job of describing their methodology [2]. However, ethicists and legal experts were not included as participants for these guidelines. Although the authors note that an expert overview of the legal and ethical framework of withdrawal of life-sustaining treatments in Canada was provided for workshop participants, the inclusion of ethicists and legal experts in the consensus process would have been beneficial [2]. Finally, there are many aspects of end-of-life care that are influenced by regional policies [8], so it is important to acknowledge that consensus achieved in one region may not apply in another. The guidelines proposed by Downar and colleagues [2] were generated by experts from a single country and may not be compatible with ethical and legal standards in other nations. When possible, multidisciplinary and international groups should be involved in the consensus process, as this may promote acceptance of guidelines on a broader scale.

\begin{abstract}
Author details
${ }^{1}$ Division of Pulmonary and Critical Care Medicine, Harborview Medical Center, University of Washington, 325 Ninth Avenue, Box 359762, Seattle, WA 98104, USA. ${ }^{2}$ Department of Intensive Care Medicine, Gelre Hospitals, Apeldoorn Location, Apeldoorn, The Netherlands. ${ }^{3}$ Department of Anesthesiology and Critical Care Medicine, Hadassah Hebrew University Medical Center, Jerusalem, Israel.
\end{abstract}

Compliance with ethical standards

\section{Conflicts of interest}

On behalf of all authors, the corresponding author states that there is no conflict of interest.

Received: 2 March 2016 Accepted: 17 March 2016

Published online: 7 April 2016

\section{References}

1. Sprung CL, Truog RD, Curtis JR, Joynt GM, Baras M, Michalsen A, Briegel J, Kesecioglu J, Efferen L, De Robertis E, Bulpa P, Metnitz P, Patil N, Hawryluck L, Manthous C, Moreno R, Leonard S, Hill NS, Wennberg E, McDermid RC, Mikstacki A, Mularski RA, Hartog CS, Avidan A (2014) Seeking worldwide professional consensus on the principles of end-of-life care for the critically ill. The consensus for worldwide end-of-life practice for patients in intensive care units (WELPICUS) study. Am J Respir Crit Care Med 190(8):855-866

2. Downar J, Delaney JW, Hawryluck L, Kenny L (2016) Guidelines for the withdrawal of life-sustaining measures. Intensive Care Med. doi:10.1007/ s00134-016-4330-7

3. Garland A, Connors AF (2007) Physicians'influence over decisions to forego life support. J Palliat Med 10(6):1298-1305

4. Sprung CL, Maia P, Bulow HH, Ricou B, Armaganidis A, Baras M, Wennberg E, Reinhart K, Cohen SL, Fries DR, Nakos G, Thijs LG (2007) The importance of religious affiliation and culture on end-of-life decisions in European intensive care units. Intensive Care Med 33(10):1732-1739

5. Wilkinson DJ, Truog RD (2013) The luck of the draw: physician-related variability in end-of-life decision-making in intensive care. Intensive Care Med 39(6):1128-1132

6. Paruk F, Kissoon N, Hartog CS, Feldman C, Hodgson ER, Lipman J, Guidet B, Du B, Argent A, Sprung CL (2014) The Durban world congress ethics round table conference report: III. Withdrawing mechanical ventilationthe approach should be individualized. J Crit Care 29(6):902-907

7. Bulow HH, Sprung CL, Baras M, Carmel S, Svantesson M, Benbenishty J, Maia PA, Beishuizen A, Cohen S, Nalos D (2012) Are religion and religiosity important to end-of-life decisions and patient autonomy in the ICU? The Ethicatt study. Intensive Care Med 38(7):1126-1133

8. Mark NM, Rayner SG, Lee NJ, Curtis JR (2015) Global variability in withholding and withdrawal of life-sustaining treatment in the intensive care unit: a systematic review. Intensive Care Med 41(9):1572-1585 